\title{
STEIN - Sistema de Tráfego e Intervenções Desenvolvimento de um Sistema de Transporte Inteligente
}

\author{
Matheus S. Quessada ${ }^{2}$, Rickson S. Pereira ${ }^{2}$, Euclydes N. Gottsfritz ${ }^{1,2}$, \\ Douglas D. Lieira ${ }^{1,2}$, Luis H. V. Nakamura ${ }^{1}$, Marco A. C. da Silva ${ }^{1}$, Rodolfo I. Meneguette ${ }^{2,3}$ \\ ${ }^{1}$ Departamento de Ciência da Computação - Instituto Federal de São Paulo (IFSP) \\ Catanduva, SP - Brasil \\ ${ }^{2}$ Departamento de Ciência da Computação - Universidade Estadual Paulista (UNESP) \\ São José do Rio Preto, SP - Brasil \\ ${ }^{3}$ Instituto De Ciências Matemáticas e de Computação - Universidade de São Paulo (USP) \\ São Carlos, SP - Brasil
}

\begin{abstract}
The increase in urban centers has several side effects, such as socioeconomic and health problems for the general population, due to the generation of toxic gases, as well as obstacles involving traffic, thus generating loss of money due to lost time. The proposed work is the development of a traffic monitoring system and the generation of information about it, such as adverse events present on the roads. For the evaluation of the proposed system, a use case was developed for the city of Catanduva-SP, which was carried out a test with the proposed systems, it is possible to verify the reduction of the vehicle downtime by $45 \%$ and a reduction of travel time of $50 \%$ with an average speed of $33 \mathrm{~km} / \mathrm{h}$.
\end{abstract}

Resumo. O aumento dos centros urbanos tem como efeitos colaterais diversos problemas, como socioeconômicos e de saúde para a população em geral, devido a geração de gases tóxicos, também como empecilhos envolvendo o trânsito, gerando assim perda de dinheiro devido ao tempo perdido. O trabalho proposto é o desenvolvimento de um sistema de monitoramento de tráfego e geração de informação sobre o mesmo, tais como eventos adversos presentes nas vias. Para avaliação do sistema proposto, um caso de uso foi desenvolvido para a cidade de Catanduva - SP, o qual foi realizado um teste com os sistemas propostos, é possível verificar a redução do tempo de parada do veículo em $45 \%$ e uma redução do tempo de viagem de $50 \%$ com uma média de velocidade de $33 \mathrm{~km} / \mathrm{h}$.

\section{Introdução}

Diversos problemas de tráfego podem ser relacionados ao constante aumento no número de veículos circulando nas cidades brasileiras. De acordo com [Souza 2019] até setembro de 2019, foram emplacados um total de 2.952.485 veículos em geral. Além desse crescimento e da falta de planejamento em cidades e grandes centros urbanos, podese acarretar diversos problemas socioeconômicos [Pereira et al. 2019, Zhong et al. 2013], como congestionamentos e problemas de saúde devido a exposição a gases tóxicos emitidos. Países como os Estados Unidos, Reino Unido e Alemanha gastaram aproximadamente uma quantia de US\$ 461 bilhões em 2017 com problemas relacionados a transporte [Cookson and Pishue 2017].

Apesar do lado negativo desse crescimento no número de veículos, aspectos positivos podem ser destacados quando há um planejamento adequado por trás disso. Esses veículos podem ser utilizados como ferramentas para realizar um monitoramento para ambientes inteligentes, utilizando recursos de bordo e de comunicação desses veículos. Dessa forma, é possível fornecer dados que são compatíveis com computação local 
[Meneguette et al. 2018, Al-Sultan et al. 2014]. Esses recursos adicionados nos veículos, podem servir como novos serviços e aplicações que serão utilizados em sistemas de transporte inteligentes com foco em aumentar a segurança e o conforto dos cidadãos, permitindo otimizar suas rotinas diárias em diversas áreas da cidade [Silva et al. 2018].

Para oferecer um sistema de transporte inteligente, foi proposto o STEIN - Sistema Tráfego E Intervenções que tem como objetivo informar aos usuários sobre as condições de tráfego da cidade em tempo real, informar as possíveis intervenções de trânsito que estão ocorrendo na cidade no momento e sugerir rotas alternativas como opção para que os usuários possam desviar dessas intervenções e escolher a melhor rota para chegar ao seu destino. Assim, o sistema planejado utilizará algoritmos de administração de fluxo de vias, informando ao usuário quais ruas estão bloqueadas ou interrompidas devido a algum tipo de evento. Neste artigo, foi considerado um evento como uma intervenção em que a administração da cidade precisa interromper o trânsito, ou seja, um trabalho de melhoria, uma obra em uma rua ou em um conjunto de bairros. O sistema dependerá da conexão da rede móvel do smartphone com um web service, sendo o acesso dos usuários realizado através ou do smarphone ou de um computador.

Portanto, a maior contribuição desse trabalho é desenvolver um sistema de transporte inteligente que fornece as seguintes características:

1. Monitoramento do tráfego da cidade;

2. Informação sobre possíveis eventos da via;

3. Sugestão de rotas alternativas em tempo real;

4. Otimizar o fluxo de veículos da cidade, minimizando o tempo de congestionamento.

Além disso, com o gerenciamento das informações de intervenções no trânsito, o STEIN possibilita que a prefeitura tenha controles dos dados de intervenções/eventos inseridos, pois devem ser autorizados. Assim, também tem os dados para obter informações de todos eventos que ocorrerem, o que não é permitido gerenciar com outras ferramentas.

O artigo está estruturado da seguinte forma: Seção 2 descreve os trabalhos relacionados. Seção 3 apresenta o desenvolvimento e o uso da arquitetura. Seção 4 apresenta os resultados de validação de um caso de uso na cidade de Catanduva, a qual a arquitetura foi implementada. Finalmente, na Seção 5 é apresentado a conclusão e trabalhos futuros.

\section{Trabalhos Relacionados}

Essa seção apresenta os trabalhos relacionados que abordam os problemas de gerenciamento de rota, gerenciando e monitorando o tráfego em centros urbanos. Nos últimos anos, diversos trabalhos exploraram o problema [Liebig et al. 2017, Lin et al. 2016, Pan et al. 2013, Quessada et al. 2019]. Entretanto, até agora, não há uma abordagem prática de serviços em tempo real que fornece uma efetiva informação para o veículo sobre o que está acontecendo nas ruas, assim como capturar informações pelos smartphones dos condutores para realizar um monitoramento eficiente e sugerindo novas rotas baseadas nas informações das ruas.

No mercado há algumas ferramentas que informam sobre as condições das vias como o Google Maps ${ }^{1}$ e o Waze ${ }^{2}$. A grande diferença entre a ferramenta proposta e essas já encontradas no mercado, é que o STEIN permite ao gerenciador controlar o cadastramento e a visualização das intervenções, permitindo se a mesma é aprovada ou não.

\footnotetext{
${ }^{1} \mathrm{https}: / /$ www.google.com.br/maps

${ }^{2}$ https://www.waze.com/pt-BR
} 
Em [Lafferriere 2019], é proposto um estudo para controle de congestionamento de tráfego urbano, utilizando uma abordagem de controle de sinais de tráfego urbano. No trabalho foi utilizado modelos matemáticos para simulação no MATLAB ${ }^{3}$, com base nos princípios de controle de consenso de rede, considerando o ciclo de mudança de trânsito de forma distribuída. No entanto, não considera a gerencia de intervenções, que podem ser informadas para evitar o congestionamento antes que aconteça.

Ao contrário dos trabalhos propostos que utilizam simulador, esse trabalho oferece um melhor serviço em tempo real que fornece informações sobre o veículo e informações capturadas através do smartphone do motorista, realizando um gerenciamento, monitoramento e sugerindo rota alternativa.

\section{STEIN - Sistema de Tráfego e Intervenções}

Nesta seção, descrevemos o sistema proposto, denominado STEIN - Sistema de Tráfego E Intervenções, que visa fornecer informações relevantes aos usuários em tempo real, para que eles possam tomar a melhor decisão de otimizar sua mobilidade em um ambiente urbano. Além disso, o STEIN pode sugerir possíveis rotas alternativas para o motorista evitar um local em que ocorra um evento. Portanto, o STEIN não apenas fornecerá informações sobre eventos que estão ocorrendo nas estradas e sugerirá rotas alternativas, mas também fornecerá monitoramento da mobilidade urbana, no qual o usuário poderá visualizar os pontos possivelmente congestionados, visando a redução de congestionamentos e o aumento de segurança e conforto para os motoristas.

O STEIN utiliza um aplicativo instalado no dispositivo móvel do condutor, onde o mesmo necessita possuir um sistema de geolocalização. Este aplicativo móvel captura informações de localização, velocidade, direção e captura as condições de tráfego da rota pré-estabelecida para executar uma possível alteração de rota, caso haja necessidade. Além disso, o sistema precisará transmitir essas informações para um centro de controle. Este centro fornecerá um conjunto de serviços para otimizar o fluxo de veículos nas vias, além disso, ele é necessário para a execução do STEIN em plena estabilidade e de maneira eficiente, visando flexibilidade e baixo custo.

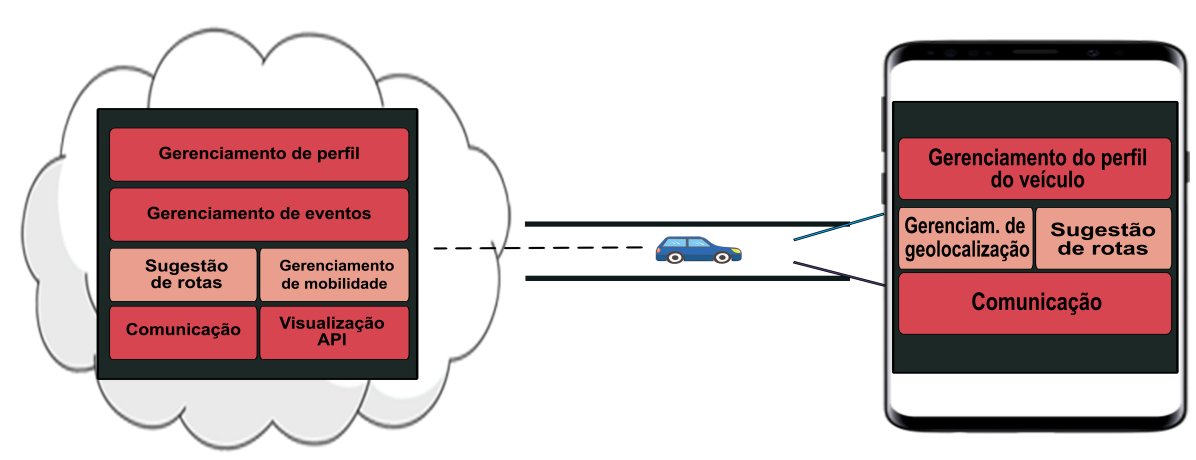

Figure 1. Visão Geral do STEIN

Os serviços em nuvem foram utilizados para manter a escalabilidade do sistema diante da demanda por solicitações de serviço. A comunicação entre a nuvem e os dispositivos móveis ocorrerá nas redes celulares de forma mais ampla. Além disso, na grande maioria dos grandes centros urbanos, a infraestrutura de rede celular cobre toda a cidade,

\footnotetext{
${ }^{3}$ https://www.mathworks.com/products/matlab.html
} 
o que significa que não haverá regiões sem conexão. Além disso, o STEIN fornecerá um mecanismo de comunicação de segurança por meio de mecanismos de criptografia para dificultar o acesso às informações de localização do motorista. Uma visão geral do sistema STEIN está disponível na seção abaixo.

O STEIN é dividido em dois componentes como se pode ver na Figura 1:

1. Cloud: consiste em todo o armazenamento e processamento de informações, roteamento, alteração de rota e monitoramento de informações do motorista. Gerencia os eventos que podem ocorrer, ou seja, realiza o registro, a disseminação e o controle dos que estão ocorrendo.

2. Dispositivo móvel: interface que irá enviar e receber informações com a nuvem sobre a localização geográfica do motorista, informações de sugestão de rota e de eventos, permitindo a tomada de decisões em tempo real pelo motorista.

Para melhor entendimento e organização, o componente da Cloud é divido em um total de seis módulos:

Gerenciamento de Perfis: é responsável pelo gerenciamento de perfil dos usuários do sistema, onde podem existir três tipos:

- Usuário: Irá apenas visualizar o mapa que contém os veículos que trafegam na cidade, em tempo real. Esse perfil também é capaz de visualizar os eventos, como interdições, e onde estão ocorrendo.

- Empresa: Além de todas as permissões do nível "Usuário", o perfil "Empresa" é capaz de solicitar à prefeitura intervenções para realizar algum reparo, obra ou melhoria na via.

- Prefeitura: Este perfil é composto por todas as permissões dos itens anteriores, além de ser capaz de aceitar ou recusar uma solicitação de interdição, criar eventos em um conjunto de ruas e registrar e alterar o perfil de novos usuários.

Gerenciamento de Eventos: Este módulo responde pelos eventos registrados no sistema, assim como dissemina essas interdições para todos os usuários. As interdições de trânsito são cadastradas por um agente da prefeitura, o qual recebe as solicitações das empresas responsáveis para obras nas vias, ou por um responsável da empresa, solicitando a autorização para a prefeitura para que a mesma conceda a liberação.

Gerenciamento de Mobilidade: Recebe as informações de tipo de veículo (carro, motocicleta, bicicleta e outros) e localização (latitude e longitude) dos motoristas. Sua tarefa também é divulgar informações em tempo real sobre o fluxo de veículos que estão em circulação nas vias da cidade, possibilitando a visualização dessa informação.

Sugestão de Rota: Esse módulo sugere rotas alternativas a fim de evitar a formação de congestionamento devido a algum evento ocorrido na rota original, isso também inclui rotas já congestionadas devido a uma alta densidade de veículos. Essa rota é sugerida com base nos campos de origem e destino, se informados pelo condutor; é feita uma requisição a Google API Directions para sugerir a rota alternativa, plotando-a no mapa.

API de Visualização: É responsável por permitir o acesso inteligível às informações contidas no sistema através de uma interface para visualiza-las e disponibiliza-las à novos serviços.

Comunicação: Responsável pela comunicação entre a cloud e o dispositivo móvel afim de prover a captura das informações solicitadas pelo serviço. É utilizado uma conexão segura encriptada através do protocolo SSL. 
Na Figura 2 pode-se ver a tela de cadastro das intervenções no site assim como as rotas interditados.

Já o dispositivo móvel, tem sua organização subdividida em quatro componentes:

Gerenciamento de Perfil do Veículo: Tem a tarefa de informar a cloud qual o tipo de veículo está iniciando aquela navegação, como por exemplo se é um carro, uma motocicleta ou uma bicicleta.

Gerenciamento de localização: É responsável por enviar as informações de latitude e longitude para a cloud no tempo estabelecido.

Sugestão de Rota: Esse módulo solicita sugestões de rota e escolhe qual delas o motorista deverá seguir.

Comunicação: Assim como no módulo de mesmo nome que consta na cloud, é responsável por manter a comunicação com a cloud e permitir o envio das informações solicitadas pelo serviço.

Uma visão de como é a tela do aplicativo pode ser vista na Figura 3.

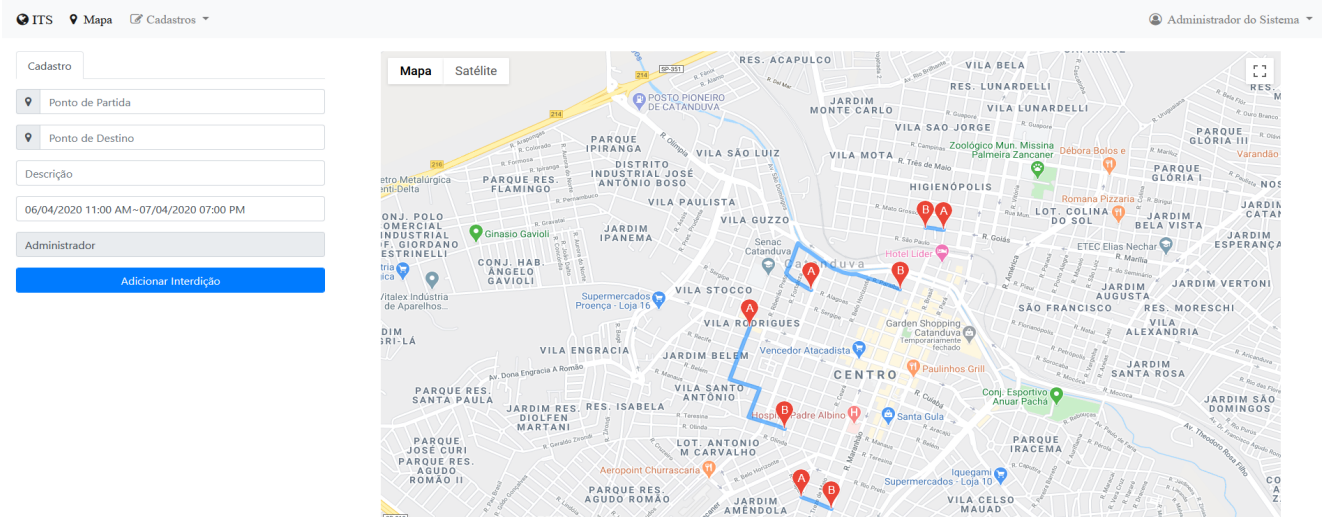

Figure 2. Visão da Tela de Cadastro de Intervenções.

\subsection{Disponibilização do código e informações gerais}

Os componentes utilizados para desenvolver o STEIN foram desenvolvidos através da licença GNU General Public License (Licença Pública Geral GNU) e se encontram disponíveis no link: http: / / rsd. ctd. ifsp.edu.br/?page_id=85. Nesse link são encontrados os códigos fonte das ferramentas, assim como tutorial e documentação.

\section{Avaliação}

Esta seção descreve a avaliação do STEIN em comparação com o padrão de mobilidade da cidade de Catanduva chamado MP (Mobilidade Padrão). O MP é o rastreamento original da mobilidade veicular, onde os veículos não têm informação sobre os eventos que estão ocorrendo nas ruas em tempo real. Isso permitirá entender os ganhos do STEIN em comparação com uma abordagem tradicional.

Para a avaliação do STEIN, consideramos toda a área urbana da cidade e foi avaliado o impacto da eficiência do tráfego utilizando o STEIN em comparação com o MP, em outras palavras, o impacto do STEIN na cidade de Catanduva.

Nessa avaliação, o objetivo é o impacto da eficiência do tráfego usando o STEIN em comparação com o MP. Para isso, foram utilizados dois mil veículos, divididos em 


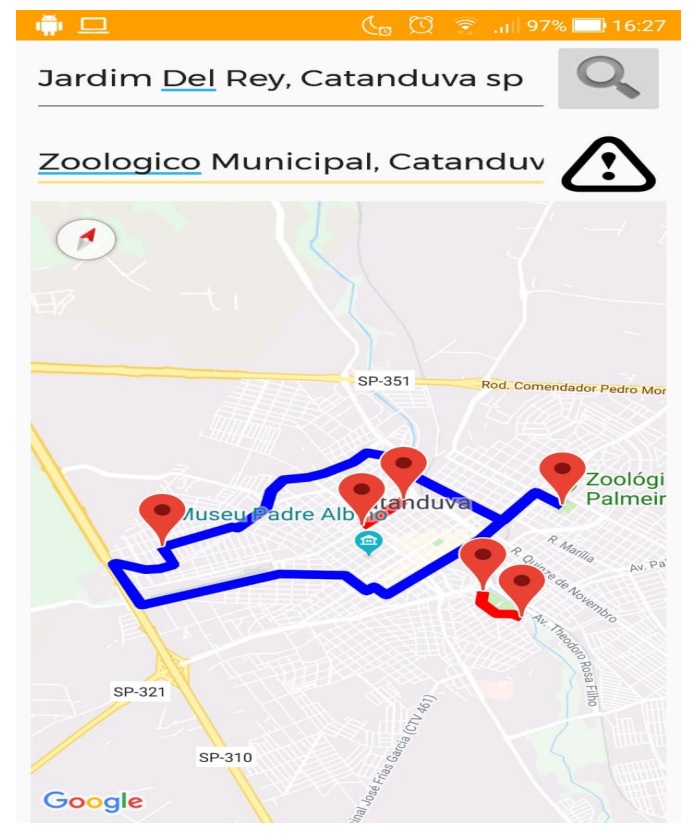

Figure 3. Tela de Rotas do Aplicativo.

dois grupos: (i) mil veículos receberam notificações de eventos, intervenções viárias; e (ii) mil não receberam essas notificações, apenas a posição geográfica dos veículos foi atualizada. Além disso, a cidade tem mais motoristas que trafegarão com os veículos que estão sendo monitorados. A impossibilidade de avaliar esses motoristas se deve à falta de uso do STEIN. Além disso, durante a avaliação, foram realizadas 3 intervenções em que duas ruas estão no centro da cidade e outra na avenida principal dela, próxima ao mercado municipal. As intervenções correspondem a melhorias das ruas nas quais precisaram ser bloqueadas ou fechadas para a execução dessa tarefa. Por exemplo, se a cidade precisar recapear uma rua para melhorar o asfalto, essa rua precisará ser fechada para que essa melhoria ocorra.

O recrutamento de usuários foi realizado através de divulgação do aplicativo móvel em meios de comunicação de massa como rádio e TV e o aplicativo foi disponibilizado através da Google Play.

O sistema foi avaliado quando a maioria dos veículos estavam em circulação, ou seja, das $11 \mathrm{~h}$ às $13 \mathrm{~h}$. É o intervalo que os motoristas almoçam e voltam ao trabalho, sendo considerado um dos horário de pico da cidade de Catanduva. Visto que a aplicação atua de forma individual, o horário de pico não interfere no desempenho da mesma, causando latência ou outro tipo de problema. As seguintes métricas foram usadas para avaliar o STEIN:

- Tempo de Viagem: foi considerado o tempo médio de viagem desde o ponto de partida até o destino de todos os veículos;

- Tempo parado: o tempo médio gasto em engarrafamentos para todos os veículos;

- Velocidade média: a velocidade média de todos os veículos;

- Distância percorrida: a distância média percorrida por todos os veículos.

A Figura 4 mostra os resultados obtidos para avaliar a eficiência do tráfego usando o STEIN. Como observado na Figura 4(a), o STEIN obteve uma redução de tempo de aproximadamente $42 \%$ em comparação com o MP. Essa redução se deve ao uso dos STEIN pelos motoristas, nesse caso eles foram avisados sobre um evento nas ruas (por 
exemplo, congestionamento ou colisão de veículos), para que possam seguir rotas alternativas e evitar a maior densidade de tráfego próximo a essas ocorrências. Esse tempo mais curto em que o veículo parou afeta diretamente o tempo de viagem, como podemos ver na Figura 4(b). O STEIN reduziu o tempo de viagem em aproximadamente $50 \% \mathrm{em}$ comparação com o MP. Outro fator que contribuiu para essa redução foi a velocidade média dos veículos.

A Figura 4(c) mostra os resultados obtidos para a velocidade média dos veículos. Como esperado, o STEIN alcançou uma maior velocidade média do veículo. Isso ocorre porque o STEIN sugere um conjunto de alternativas de maior facilidade de locomoção para o motorista decidir qual delas seguir. Essa sugestão leva em consideração as ruas interditadas e também a densidade em toda a rota, evitando assim o surgimento de um novo congestionamento em outra estrada.

Analisando a distância percorrida Figura 4(d), podemos ver que o MP diminui a distância percorrida porque o motorista não precisou desviar de sua rota original para chegar ao destino. Em outras palavras, o STEIN obteve um aumento de aproximadamente $1 \mathrm{~km}$ devido ao desvio na rota do veículo para evitar o evento.

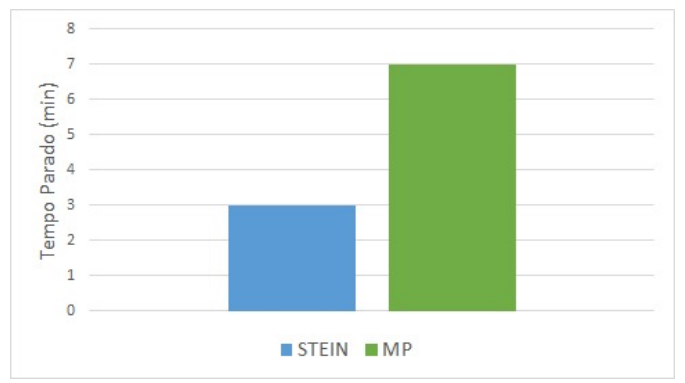

(a) Tempo Parado.

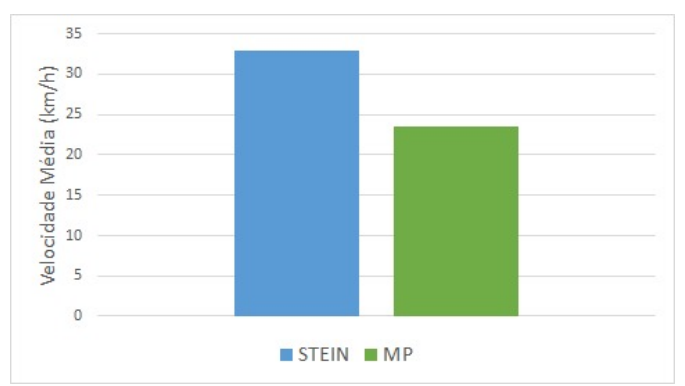

(c) Média de Velocidade

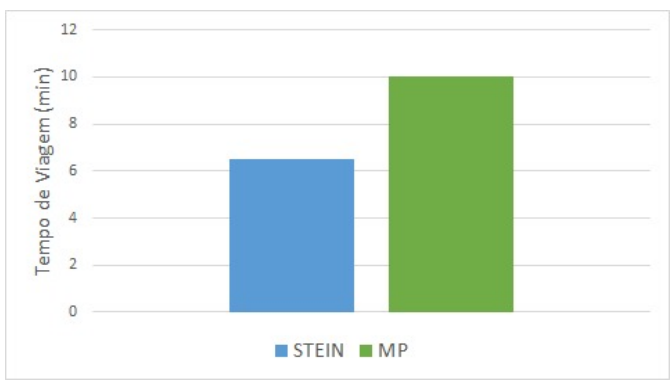

(b) Tempo de Viagem.

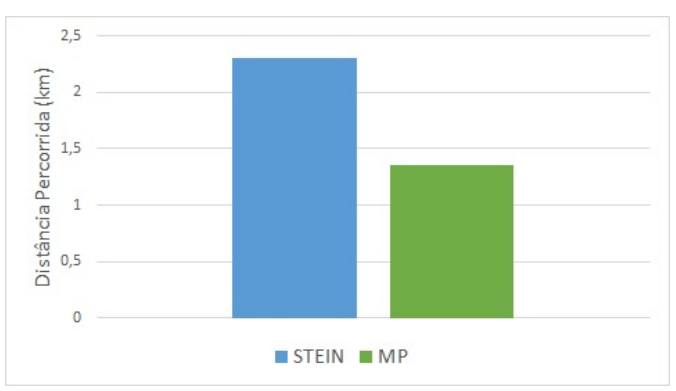

(d) Distância Percorrida.

Figure 4. Impacto do STEIN no sistema de transporte.

Portanto, o STEIN alcançou uma redução no tempo que o veículo esteve parado devido a um evento ou congestionamento. Consequentemente, reduziu o tempo de viagem e aumentou a velocidade média do veículo. Em contrapartida houve um aumento na distancia percorrida devido ao desvio.

\section{Conclusão}

O presente trabalho apresenta o desenvolvimento e as funcionalidades de um sistema de transporte inteligente que permite o rastreio e a rastreabilidade de veículos, permitindo aos motoristas serem informados sobre possíveis eventos que estão ocorrendo na via.

O dispositivo móvel atualiza a central em tempo real com o posicionamento desses usuários em tempo real através de mecanismos de geolocalização, assim como receber 
os pontos de eventos que estão ocorrendo na cidade e as rotas alternativas para que os motoristas possam desviar.

Como resultado, pode-se ver uma redução do tempo de parada gasto durante um evento ou congestionamento. Além disso, foi reduzido o tempo de viagem e aumentada a velocidade média do veículo.

Como trabalhos futuros, espera-se desenvolver novos serviços de informação, como implementar mensagens de alerta ao usuário quando tiver alguma intervenção em sua rota. Também pode-se implementar um módulo para o órgão responsável encaminhar mensagens aos usuários do aplicativo. Além disso, planeja-se aplicar o STEIN em outros diferentes contextos, como ambientes rodoviários.

\section{Referências}

Al-Sultan, S., Al-Doori, M. M., Al-Bayatti, A. H., and Zedan, H. (2014). A comprehensive survey on vehicular ad hoc network. Journal of network and computer applications, 37:380-392.

Cookson, G. and Pishue, B. (2017). Inrix global traffic scorecard-appendices. INRIX research.

Lafferriere, G. (2019). Traffic signal consensus control. NITC-RR-1165. Portland, OR: Transportation Research and Education Center (TREC).

Liebig, T., Piatkowski, N., Bockermann, C., and Morik, K. (2017). Dynamic route planning with real-time traffic predictions. Information Systems, 64:258-265.

Lin, J., Yu, W., Yang, X., Yang, Q., Fu, X., and Zhao, W. (2016). A real-time en-route route guidance decision scheme for transportation-based cyberphysical systems. IEEE Transactions on Vehicular Technology, 66(3):2551-2566.

Meneguette, R. I., De Grande, R., and Loureiro, A. A. (2018). Intelligent Transport System in Smart Cities. Springer.

Pan, J., Popa, I. S., Zeitouni, K., and Borcea, C. (2013). Proactive vehicular traffic rerouting for lower travel time. IEEE Transactions on vehicular technology, 62(8):35513568 .

Pereira, R. S., Lieira, D. D., da Silva, M. A. C., Pimenta, A. H. M., da Costa, J. B. D., Rosário, D., and Meneguette, R. I. (2019). A novel fog-based resource allocation policy for vehicular clouds in the highway environment. In 2019 IEEE Latin-American Conference on Communications (LATINCOM), pages 1-6.

Quessada, M., Cristiani, A., Ranzani, P., Pereira, R., Leal, M., and Meneguette, R. (2019). Desenvolvimento de um modelo de mobilidade urbana em tempo real para simuladores de rede. In Anais Estendidos do XXXVII Simpósio Brasileiro de Redes de Computadores e Sistemas Distribuídos, pages 81-88, Porto Alegre, RS, Brasil. SBC.

Silva, B. N., Khan, M., and Han, K. (2018). Towards sustainable smart cities: A review of trends, architectures, components, and open challenges in smart cities. Sustainable Cities and Society, 38:697-713.

Souza, L. (2019). Vendas de veículos crescem 11,4\% no acumulado até setembro. Disponível em: http://agenciabrasil.ebc.com.br/economia/noticia/2019-10/vendas-deveiculos-crescem-114-no-acumulado-ate-setembro.

Zhong, R., Sumalee, A., Pan, T., and Lam, W. (2013). Stochastic cell transmission model for traffic network with demand and supply uncertainties. Transportmetrica A: Transport Science, 9(7):567-602. 